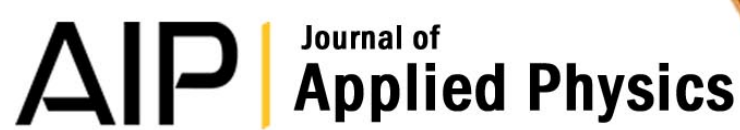

\section{Light amplification and lasing in a (meta-phenylene vinylene) copolymer}

Hacene Manaa, Fryad Z. Henari, Ahmed Al-Saie, Anna Drury, Takeyuki Kobayashi et al.

Citation: J. Appl. Phys. 93, 1871 (2003); doi: 10.1063/1.1537463

View online: http://dx.doi.org/10.1063/1.1537463

View Table of Contents: http://jap.aip.org/resource/1/JAPIAU/v93/i4

Published by the American Institute of Physics.

\section{Additional information on J. Appl. Phys.}

Journal Homepage: http://jap.aip.org/

Journal Information: http://jap.aip.org/about/about_the_journal

Top downloads: http://jap.aip.org/features/most_downloaded

Information for Authors: http://jap.aip.org/authors

\section{ADVERTISEMENT}

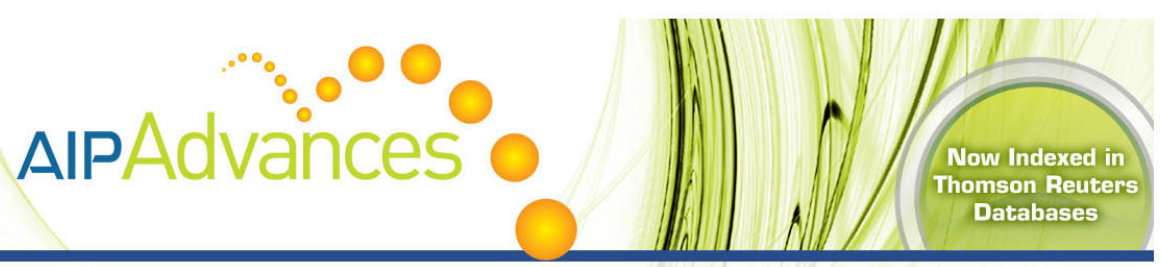

\section{Explore AIP's open access journal: Rapid publication \\ - Article-level metrics \\ - Post-publication rating and commenting}




\title{
Light amplification and lasing in a (meta-phenylene vinylene) copolymer
}

\author{
Hacene Manaa, Fryad Z. Henari, ${ }^{a)}$ and Ahmed Al-Saie \\ Department of Physics, University of Bahrain, P.O. Box 32038, Isa Town, Kingdom of Bahrain
}

Anna Drury, Takeyuki Kobayashi, and Werner J. Blau

Materials Ireland Polymer Research Center, Department of Physics, Trinity College Dublin, Dublin 2, Ireland

(Received 5 July 2002; accepted 18 November 2002)

\begin{abstract}
We report on single pass optical gain and lasing measurements on a blue-emitting copolymer, poly ( $m$-phenylene vinylene-co-2,5-dioctyloxy-p-phenylene vinylene) under $0.5 \mathrm{~ns}$ pulsed photoexcitation. This soluble copolymer is designed and synthesized for light amplification and lasing applications. Blue photoluminescence is achieved by introduction of the meta linkage of the phenylene rings in the polymer backbone, which reduces the extent of delocalization of $\pi$ electrons. The large Stokes shift of about $70 \mathrm{~nm}$ implies a low reabsorption rate, and leads to easier creation of population inversion and hence to efficient light amplification. A solution of the polymer exhibits amplified spontaneous emission evidenced by spectral narrowing and a superlinear increase of output intensity when it is photoexcited at $337 \mathrm{~nm}$. By means of the variable excitation stripe length method, net gains of 18 and $24 \mathrm{~cm}^{-1}$ are deduced for different pump energies of 71 and $113 \mu \mathrm{J}$, respectively. Moreover, laser emission from the polymer solution is obtained in a simple cavity formed by Fresnel reflections at the cuvette-solution interfaces. A conversion efficiency as high as $27 \%$ and a wide tuning range between 470 and $492 \mathrm{~nm}$ in solutions are demonstrated. (C) 2003 American Institute of Physics. [DOI: 10.1063/1.1537463]
\end{abstract}

\section{INTRODUCTION}

Organic conjugated polymers have become important materials due to their potential as an active medium in light emitting diodes ${ }^{1,2}$ and electro-luminescent displays. ${ }^{3}$ The high photoluminescence quantum yield of many conjugated polymers makes them promising candidates for active laser media and, in fact, optical gain and lasing have been demonstrated in many conjugated polymers both in solutions and films. ${ }^{4-8}$ The advantage of these materials is that the lasing spectrum covers wide regions in the visible that are still not easily accessible by the most commonly used inorganic semiconductor materials. Furthermore, the tolerance of conjugated polymers to high concentrations of emissive species without alteration of their optical properties and degradation of laser performance is a potential advantage over classical organic dyes. The high concentration of emissive species together with the large emission cross section induced due to the fully allowed transition between $S_{0}$ and $S_{1}$ states is responsible for the large optical gain inherent in this class of materials. Ultrafast pump-probe measurements of thin films have demonstrated very large optical gains of the order of $10^{4} \mathrm{~cm}^{-1}$ in some conjugated polymers. ${ }^{9}$

Conjugated polymers such as poly( $p$-phenylene vinylene) (PPV) have large vibronic fluorescence bands in the visible spectral range with large stimulated emission cross sections of the order of $10^{-16} \mathrm{~cm}^{2} .{ }^{10}$ By modifying the structure of PPV various luminescent polymers can be made which can be used to fabricate devices that emit colors from the UV to the IR regions. ${ }^{11}$ For example, introducing electron-donating groups onto the polymer unit leads to a

\footnotetext{
a)Electronic mail: fryad@sci.uob.bh
}

redshift in the absorption maxima ${ }^{12}$ and introducing long alkoxy chains increases the solubility and thus the processability of the polymer. ${ }^{13}$ Blueshifted luminescence can be achieved by reducing the effective conjugation of the main polymer chain. One way of doing this is by incorporating meta-linked phenyl rings within the backbone; ${ }^{14}$ for example, multilayer electroluminescent devices (LEDs) have been made from the derivative poly $(m$-phenylene vinyleneco-2,5-dioctyloxy-p-phenylene vinylene) (DOP-PmPV). ${ }^{15}$ Currently studies are in progress to understand the process of emission and light amplification in such materials.

Here we report the optical gain measurements of DOPPmPV in toluene. The spectral narrowing and the exponential increase of the output intensity upon an increase of the excited stripe length clearly show the existence of optical gain by stimulated emission. Furthermore, we demonstrate laser emission from DOP-PmPV in toluene solutions that shows that laser action can be obtained with low absorbed energy threshold.

\section{EXPERIMENTS AND RESULTS}

The copolymer is made by a Horner-Emmons condensation reaction in toluene described elsewhere. ${ }^{16}$ Full optical characterization of the copolymer was undertaken and it is given in Ref. 17. The chemical structure is shown in the inset of Fig. 1. The absorption and spontaneous emission spectra of the copolymer at room temperature are shown in Fig. 1. The absorption spectrum shows two vibronic peaks $\lambda=350$ and $412 \mathrm{~nm}$ while the emission peak $\lambda_{\max }$ occurs at $480 \mathrm{~nm}$. The two vibronic peaks correspond to the phonon-assisted optical transition connecting the $S_{0}$ electronic ground state and the first excited singlet state $S_{1}$. These optical transitions 


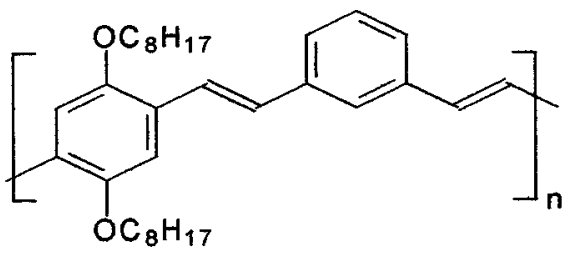

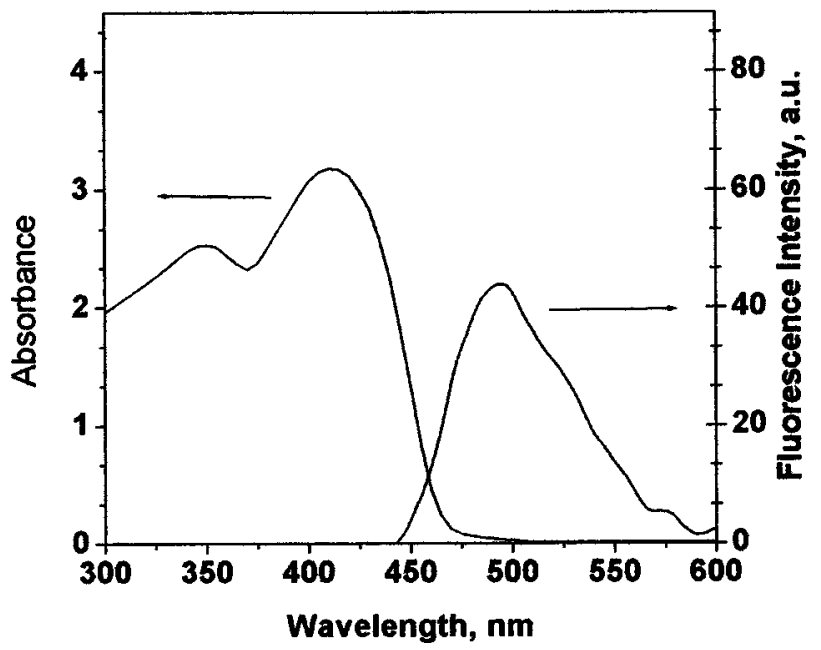

FIG. 1. Chemical structure and the absorption/emission spectra of the metaphenylene vinylene copolymer at room temperature.

can be efficiently induced with output wavelengths of either a frequency-tripled $\mathrm{Nd}^{3+}$ :YAG laser or of a $\mathrm{N}_{2}$ laser. Solutions with concentrations of 2 and $4 \mathrm{~g} / \mathrm{l}$ were prepared in toluene, and placed in a $10 \mathrm{~mm}$ cuvette. In the present study, the samples were photoexcited at $337 \mathrm{~nm}$ with a $\mathrm{N}_{2}$ laser (0.5 ns pulse width, $10 \mathrm{~Hz}$ repetition rate).

The experimental method for optical gain measurement uses a variable excitation stripe length method similar to that used by Shaklee and Leheny and by Sorek et al. ${ }^{18,19}$ It involves the detection of the single pass amplified light emitted along the length of an optically pumped sample. This measurement allows assessment of the optical gain and the laser potential in the materials without construction of a resonant cavity. The excitation beam from the $\mathrm{N}_{2}$ laser was focused onto the surface of the cuvette by a cylindrical lens of $f$ $=10 \mathrm{~cm}$, and produced a thin stripe on the cuvette approximately $0.1 \mathrm{~mm}$ in thickness and $10 \mathrm{~mm}$ in width. The sample was mounted on a translation stage to allow variation of the excited stripe length. The spontaneous fluorescence emitted in parallel to the stripe was collected and focused with a lens at the entrance of a 1200 grooves $/ \mathrm{mm}$ monochromator prior to detection with a sensitive silicon photodiode. The signal was then amplified and averaged with a boxcar averager.

Figure 2 shows the change in the fluorescent spectrum at maximum pump intensity of $2.3 \times 10^{6} \mathrm{~W} / \mathrm{cm}^{2}$, with an increase in excitation length. The amplified spontaneous emission spectrum produces a strong peak located at $480 \mathrm{~nm}$, which becomes narrower with an increase in excitation length. Figure 2 illustrates that, as the length of the excited region increases from $x=0.1$ to $0.6 \mathrm{~cm}$, i.e., by a factor of 6 , the fluorescence intensity at its peak $(480 \mathrm{~nm})$ increases by a factor of around 40 . This behavior indicates clearly that the

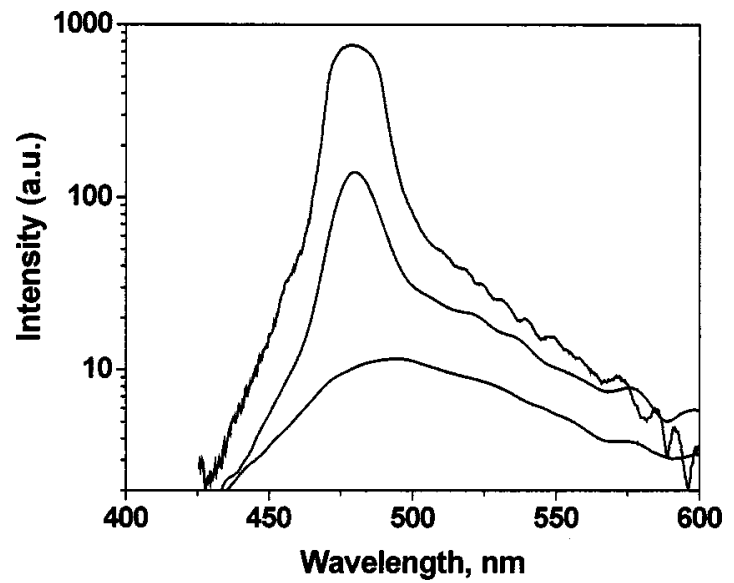

FIG. 2. Emission spectra of the copolymer, measured with three different excited region lengths. (From top to bottom: $x=0.6,0.3$ and $0.1 \mathrm{~cm}$ ).

fluorescence spectra measured are the superposition of spontaneous fluorescence and stimulated emission. While the spontaneous fluorescence intensity increases linearly with the excited region length, the stimulated emission intensity increases exponentially and narrows the global emission spectrum around its peak, where the net gain is at its maximum.

Figure 3 shows the output intensity of the emission maximum as a function of the excitation strip length for two different pump intensities. Figure 3 clearly shows a superlinear increase. The increase is a direct indication of the presence of positive gain and of the predominance of a stimulated emission mechanism. The measurements were performed on excited strip lengths up to $6 \mathrm{~mm}$. However, in order to exclude emission from inhomogeneously excited regions or the saturated regions, only the first $3 \mathrm{~mm}$ was considered for calculations.

In order to deduce the net optical gain coefficients with variation of the excitation lengths, we use an equation given by other authors: ${ }^{18,19}$

$$
I(x)=\left(I_{p} A / g_{\text {eff }}\right)\left[\exp \left(g_{\text {eff }} x\right)-1\right] .
$$

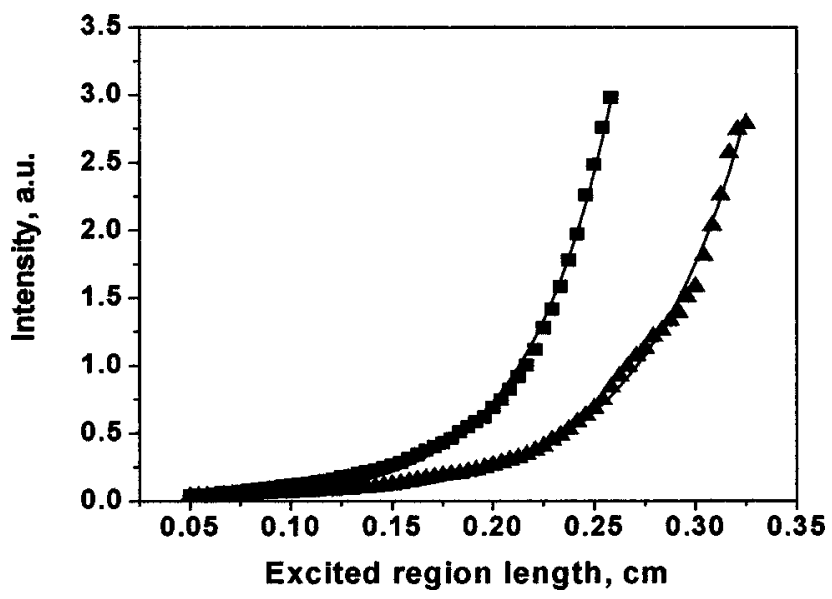

FIG. 3. Emission intensity of the copolymer as a function of the length of the excited region for two different pumping energies (squares: 113; triangles: $71 \mu \mathrm{J} /$ pulse). 


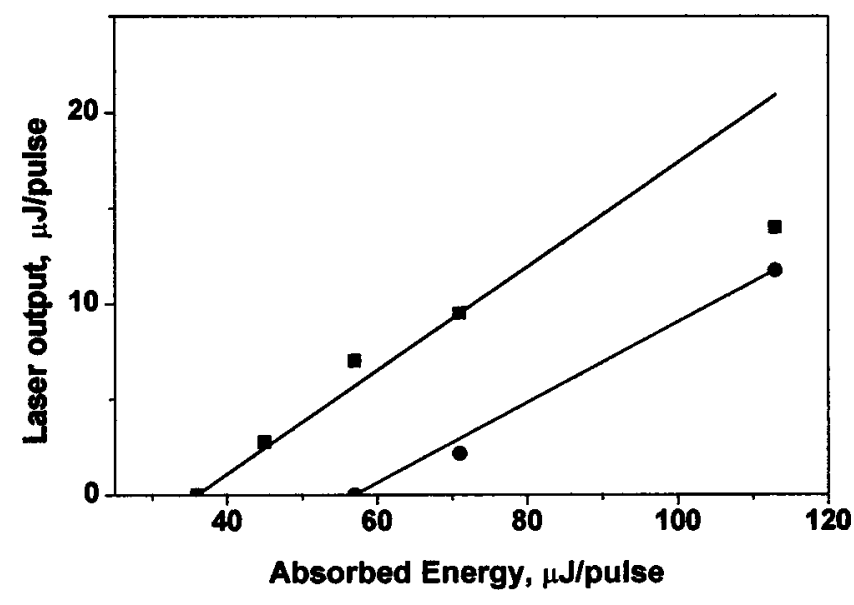

FIG. 4. Laser energy output of the copolymer as a function of the energy absorbed for different concentrations (squares: 4; circles: $2 \mathrm{~g} / \mathrm{l}$ ).

Here $I(x)$ denotes the emission intensity. The term $\mathrm{Ai}_{p}$ describes the spontaneous emission, which is proportional to the pump intensity $I_{p} . g_{e f f}=g_{s e}-\alpha$ is the net optical gain coefficient where $g_{s e}$ is the internal gain and $\alpha$ is the total optical loss per unit length in the sample. The loss is not only due to self-absorption but also due to excited state absorption. The fit of Eq. (1) to the experimental data with two adjusting parameters $g_{\text {eff }}$ and $A I_{p}$ gives $g_{\text {eff }}=18 \mathrm{~cm}^{-1}$ at a pumping energy of $71 \mu \mathrm{J}$ and $g_{\text {eff }}=24 \mathrm{~cm}^{-1}$ at a pumping energy of $113 \mu \mathrm{J} /$ pulse.

Laser action was observed when the polymer solution was pumped at $337 \mathrm{~nm}$ with the same $\mathrm{N}_{2}$ laser. The solution was put into a $1 \mathrm{~cm}$ wide quartz cuvette. The input and output pulse energies of the laser were measured using a calibrated pyroelectric detector. A monochromator was used to determine the tuning range. Laser oscillation was established to be due to Fresnel reflections between the internal faces of the cuvette. The input/output energy curve is shown in Fig. 4 for two different concentrations. We can see clear threshold behavior followed by a linear increase of output energy, which is an unambiguous feature of the onset of laser action. The spectral half width was $7 \mathrm{~nm}$. The plots show a conversion efficiency of $27 \%$ and $21 \%$ for 4 and $2 \mathrm{~g} / \mathrm{l}$, respectively. The absorbed energy threshold decreases from 57 $\mu \mathrm{J} /$ pulse for a $2 \mathrm{~g} / \mathrm{l}$ concentration to $36 \mu \mathrm{J} /$ pulse for a $4 \mathrm{~g} / \mathrm{l}$ concentration. This indicates that increasing the concentration is not necessarily accompanied by an increase of optical losses. Figure 5 shows the stimulated emission spectrum of the polymer in relaxed mode. The lasing spectrum covers a wide range from 470 to $492 \mathrm{~nm}$ with the output maximum at $480 \mathrm{~nm}$.

\section{CONCLUSIONS}

We have investigated the properties of a meta-phenylene vinylene copolymer as a lasing material. Single pass gain measurements have revealed that the copolymer in toluene solution has a net gain of $18-24 \mathrm{~cm}^{-1}$ at $480 \mathrm{~nm}$, which suggests that the material would be useful as a laser medium. Furthermore, we have demonstrated laser action in solutions

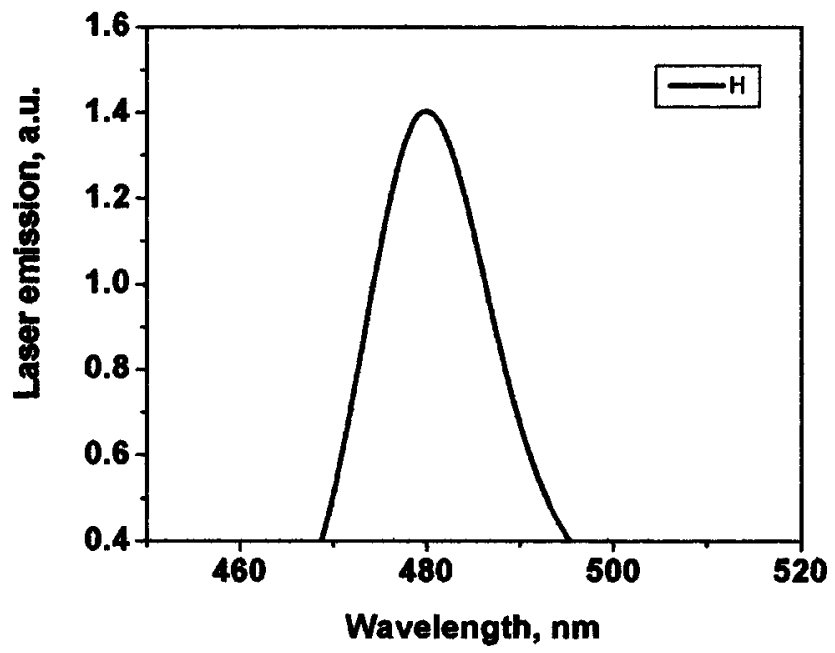

FIG. 5. Stimulated emission spectrum of the copolymer.

of the copolymer in a simple resonator defined by the cuvette walls. The high gain of the material allows laser action to occur in such a low- $Q$ cavity. The tuning range and conversion efficiency were presented. Further studies in progress include the optimization of lasing conditions and optical characterization of the material in solid-state hosts.

\section{ACKNOWLEDGMENT}

The authors would like to thank Dr Ahmad Taha of the Chemistry Department, College of Science, University of Bahrain, for valuable discussions.

${ }^{1}$ J. H. Burrough, D. D. C. Bradley, A. R. Brown, R. N. Marks, K. Mackay, R. H. Friend, and P. L. Holmes, Nature (London) 347, 539 (1990).

${ }^{2}$ N. C. Greenham and R. H. Friend, Solid State Phys. 49, 1 (1995).

${ }^{3}$ M. Berggren, G. Gustafsson, O. Inganas, M. R. Andersson, T. Hjertberg, and O. Wennerstorm, J. Appl. Phys. 76, 7530 (1994).

${ }^{4}$ F. Z. Henari, K. P. Kretsch, and W. J. Blau, Photonics Sci. News 4, 5 (1998).

${ }^{5}$ W. Holzer, A. Penzkofer, S. H. Gong, A. P. Davey, and W. J. Blau, Opt. Quantum Electron. 29, 713 (1997), and references therein.

${ }^{6}$ T. Kobayashi and W. J. Blau, Electron. Lett. 38, 67 (2002).

${ }^{7}$ W. Hollzer, A. Penzkofer, S.-H. Gong, A. Bleyer, and D. C. Bradley, Adv. Mater. 8, 974 (1996).

${ }^{8}$ N. D. Kumar, J. D. Bhawalker, P. N. Prasad, F. E. Karasz, and B. Hu, Appl. Phys. Lett. 71, 999 (1997).

${ }^{9}$ T. Virgili, D. G. Lidzey, D. D. C. Bradley, G. Cerullo, S. Stagira, and S. De Silvestri, Appl. Phys. Lett. 74, 2767 (1999).

${ }^{10}$ F. Z. Henari, H. Manna, K. P. Kretsch, W. J. Blau, H. Rost, S. Pfeiffer, A. Teuschel, H. Tillmann, and H. Horhold, Chem. Phys. Lett. 307, 163 (1999).

${ }^{11}$ Handbook of Conducting Polymers, edited by T. A. Skotheim, R. L. Elsenbaumer, and J. R. Reynolds (Decker, New York, 1998).

${ }^{12}$ J. L. Segura, Acta Polym. 49, 319 (1998).

${ }^{13}$ S. H. Askari, S. D. Rughooputh, and F. Wudl, Synth. Met. E129, 29 (1998).

${ }^{14}$ Y. Pang, J. Li, B. Hu, and F. E. Karasz, Macromolecules 31, 6730 (1998).

${ }^{15}$ D. O'Brien, A. Bleyer, D. G. Lizey, and D. D. Bradley, J. Appl. Phys. 82, 5 (1999).

${ }^{16}$ A. Drury, S. Maier, A. P. Davey, A. B. Dalton, J. N. Coleman, H. J. Byrne, and W. J. Blau, Synth. Met. 119, 151 (2000).

${ }^{17}$ A. B. Dalton, J. N. Coleman, M. in het Panhuis, B. McCarthy, A. Drury, W. J. Blau, B. Paci, J. M. Nunzi, and H. J. Byrne, J. Photochem. Photobiol., A 144, 31 (2001).

${ }^{18}$ K. L. Shaklee and R. F. Leheny, Appl. Phys. Lett. 18, 475 (1971).

${ }^{19}$ Y. Sorek, R. Reisfeld, I. Finkelstein, and S. Ruschin, Appl. Phys. Lett. 66, 1169 (1995). 